Dom. Cien., ISSN: 2477-8818

Vol. 4, núm. 1, enero, 2018, pp. 322-331

\title{
Lactancia materna. Su influjo en las malas oclusiones en niños escolares
}

\author{
Breastfeeding. Its influence on bad occlusions in school children
}

\section{Lactancia materna. Su influjo en las malas oclusiones em niños escolares}

\author{
Alba M. Mendoza-Castro ${ }^{\mathrm{I}}$ \\ lbamariamc@hotmail.com \\ Evelyn M. Tovar-Moreira II \\ evelyn.tovar@uleam.edu.ec \\ Iván Robles-Quezada ${ }^{\mathrm{III}}$ \\ ivan-robles@hotmail.com
}

Recibido: 31 de julio de 2017 * Corregido: 27 de septiembre de 2017 * Aceptado: 31 de noviembre de 2017

I. Magister en Gerencia de Salud Para el Desarrollo Local, Odontóloga Especialista en Endodoncia, Odontóloga, Docente de la Universidad Laica Eloy Alfaro de Manabí, Manta, Ecuador.

II. Magister en Gerencia Y Auditoria de Servicios de Salud Bucal, Especialista en Odontología Infantil, Doctor en Odontología, Docente de la Universidad Laica Eloy Alfaro de Manabí, Manta, Ecuador.

III. Facultad Ciencias Médicas de la Universidad Laica Eloy Alfaro de Manabí, Manta, Ecuador. 


\title{
Resumen
}

Se realizó un estudio observacional de tipo transversal. La población de estudio consistió en 202 historias clínicas (anamnesis y examen clínico) de niños de 5 a 6 años de edad, que acudieron a la consulta odontopediatría en la consulta privada en el periodo enero de 2016 hasta enero de 2017, con la finalidad de determinar la relación de la lactancia materna en la aparición de mal oclusiones. En la serie predominaron el sexo femenino, la lactancia materna combinada, así como la lengua protáctil y la succión digital como hábitos bucales deformantes. A manera de colofón, los resultados obtenidos indican una relación entre el tiempo de amamantamiento menor a 6 meses y el desarrollo de hábitos deformantes. Tienen mayor riesgo de padecer estos hábitos los niños que no recibieron lactancia o que la tuvieron solo durante periodos cortos.

Palabras clave: niños; lactancia materna; hábitos bucales deformantes; malas oclusiones.

\begin{abstract}
An observational cross-sectional study was performed. The study population consisted of 202 clinical histories (anamnesis and clinical examination) of children from 5 to 6 years of age who attended the pediatric dentistry consultation in the private clinic between January 2016 and January 2017, in order to to determine the relation of breastfeeding to the appearance of malocclusions. In the series predominated the female sex, the combined breastfeeding, as well as the protáctil language and the digital suction as deforming oral habits. As a conclusion, the results obtained indicate a relationship between the time of suckling less than 6 months and the development of deforming habits. Children who did not receive breastfeeding or who had it only for short periods are at greater risk for these habits.
\end{abstract}

Keywords: children; breastfeeding; deforming oral habits; bad occlusions.

\section{Resumo}

Se realizó um estudo observacional de tipo transversal. A população de estudo consistió em 202 historias clínicas de crianças de 5 a 6 anos de idade, que informou a consultoria odontopediatría na consulta privada em janeiro de 2016 até janeiro de 2017, com a finalidade de determinar a relação da lactância materna na aparição de mal oclusiones. Na série predominantemente o sexo feminino, a lactância materna combinada, assim como a língua protáctil e a suç̧ão digital como hábitos bucales deformantes. A forma de colofão, os resultados obtidos em uma relação entre o tempo de 
amamantamento menor a 6 meses e o desenvolvimento de hábitos deformantes. Tienen mayor risco de padecer estes hábitos, as crianças que não receberam lactância ou o mesmo durante periodos cortos.

Palavras-chave: niños; lactancia materna; hábitos bucales deformantes; malas oclusiones.

\section{Introducción}

En marzo 2004 la Organización Mundial de la Salud (OMS) y el Fondo de las Naciones Unidas para la Infancia (U.N.I.C.E.F) presentaron la "Estrategia Mundial para la Alimentación del Lactante y del Niño Pequeño", destacando a la lactancia materna como fundamental para la supervivencia infantil ${ }^{1}$. Es indudable que el amamantamiento, es la medida más eficaz y menos costosa para evitar la desnutrición y las enfermedades infecciosas durante los primeros meses de vida. Por otra parte, estudios odontológicos realizados en la última década tienden a indicar que la falta de la lactancia materna o un período corto de ésta se asocia con la presencia de mal oclusiones dentales ${ }^{2}$.

El hecho de alimentar al niño con biberón, ha demostrado que produce un desbalance muscular, debido a una diferencia fisiológica entre la forma del pezón natural y el chupete clásico ${ }^{3}$.

Durante el amamantamiento, el bebé debe presionar con la lengua los senos lactíferos para obtener la leche; pero cuando se alimenta mediante el biberón, no necesita esfuerzo alguno para darle forma a la tetilla dentro de su boca. ${ }^{2} \mathrm{Si}$ el niño succiona y regula el fluido con la lengua, la reacción de estrés normal puede dar inicio a otros hábitos como el de deglución atípica ${ }^{3}$.

De manera ideal, se impone brindar a las madres asesoramiento dietético inmediatamente después del nacimiento del niño, ya que es mejor establecer buenos hábitos que modificar los malos. Los movimientos de la lengua y la actividad incrementada del conjunto de músculos suprahiodeos, desempeñan una importante función en los períodos de succión durante el amamantamiento, puesto que sientan las bases para un mejor crecimiento y desarrollo de los maxilares ${ }^{4,5,3}$.

Una oclusión normal se refiere a la correcta relación existente entre el maxilar superior y la mandíbula, que está directamente relacionada con el crecimiento armónico de ambos maxilares y sus estructuras. Cuando se presenta un trastorno en el proceso de crecimiento y desarrollo craneofacial y del sistema estomatognático, se está en presencia de una mal oclusión ${ }^{6,7}$. 
Las alteraciones en el desarrollo de la oclusión están influenciadas por factores hereditarios, congénitos, constitucionales y ambientales. También se asocian con la presencia de caries dentales, insuficiencia respiratoria nasal, hábitos nocivos de succión, deglución, masticación y un corto periodo de lactancia materna. Estas son responsables de algunas formas de mal oclusión en la infancia, de las cuales se ha evidenciado como las más prevalentes: la mordida cruzada posterior y la mordida abierta. Estas se ven influenciadas por periodos cortos de lactancia materna ${ }^{8}$.

Por la forma en que repercute la lactancia materna en el crecimiento y desarrollo de las estructuras craneofaciales, ya que se produce una excitación de la musculatura oro facial y se estimula el desarrollo funcional y armonioso del sistema estomatognático Nos sentimos motivados para realizar el presente trabajo

\section{Materiales y método}

Se realizó un estudio observacional de tipo transversal. La población de estudio consistió en 202 historias clínicas (anamnesis y examen clínico) de niños de 5 a 6 años de edad que acudieron a la consulta odontopediatría en la consulta privada en el periodo de enero de 2016 hasta enero de 2017, con la finalidad de determinar la relación de la lactancia materna en la aparición de mal oclusiones.

Entre las variables analizadas figuraron: edad, sexo, lactancia materna, presencia actual de hábitos orales deformantes como onicofagia, succión digital, bruxismo, respiración bucal, lengua pro táctil entre otros y las mal oclusiones como mordida abierta, mordida profunda y cruzada posterior unilateral. Se utilizó el porcentaje como medida de resumen.

\section{Resultados}

Del total de pacientes (tabla 1), solo 72 (35.6\%) recibieron lactancia materna exclusiva durante al menos 3 meses; mientras que la mayoría, la combinada (58.4\%), en ambos grupos de edades. 
Tabla 1. Lactancia materna según edad

\begin{tabular}{|l|c|c|c|c|c|c|}
\hline \multirow{2}{*}{ Lactancia } & \multicolumn{3}{|c|}{ Edad } & \multicolumn{2}{c|}{ Total } \\
\cline { 2 - 7 } & \multicolumn{2}{|c|}{5 años } & \multicolumn{2}{c|}{6 años } & \multicolumn{2}{c|}{} \\
\cline { 2 - 7 } & No & $\%$ & No & $\%$ & No & $\%$ \\
\hline Exclusiva & 50 & 40.2 & 22 & 27.5 & 72 & 35.6 \\
\hline Combinada & 68 & 33.3 & 50 & 62.5 & 118 & 58.4 \\
\hline No recibió & 4 & 3.2 & 8 & 10 & 12 & 5.9 \\
lactancia & & & & & & \\
\hline Total & 122 & 60.3 & 80 & 39.6 & 202 & 100 \\
\hline
\end{tabular}

En cuanto al sexo, existió predominio del sexo femenino (58.4\%), también se aprecia el predominio de la lactancia materna combinada.

Del mismo modo, la tabla 2 muestra el número de pacientes en relación con el tiempo de consumo del biberón. El mayor porcentaje se encontró entre quienes consumieron por más de 24 meses.

Tabla 2. Tiempo de consumo de biberón

\begin{tabular}{|l|c|c|}
\hline \multicolumn{1}{|c|}{ Tiempo de Biberón } & No de paciente & $\%$ \\
\hline $1-12$ meses & 53 & 26.2 \\
\hline $13-24$ meses & 59 & 29.2 \\
\hline Más de 24 meses & 89 & 44.0 \\
\hline Nunca & 10 & 4.9 \\
\hline
\end{tabular}


La tabla 3, muestra los hábitos deformantes identificados. Al respecto la succión digital y lengua pro táctil (19.8\% y 14.8\% respectivamente) fueron los más frecuentes, así como otros hábitos. El 4.9\% no presentaron ninguna habito deformantes.

Tabla 3. Lactancia materna y tipos de hábitos deformantes

\begin{tabular}{|l|c|c|}
\hline \multicolumn{1}{|c|}{$\begin{array}{c}\text { Tipos de Hábitos } \\
\text { deformantes }\end{array}$} & No de paciente \\
\hline Onicofagia y succión & 26 & 12.8 \\
digital & 12 & 5.9 \\
\hline Onicofagia & 10 & 4.9 \\
\hline Bruxismo & 32 & 19.8 \\
\hline Succión digital & 8 & 3.9 \\
\hline Respirador bucal & 30 & 14.8 \\
\hline Lengua pro táctil & 3 & 35.1 \\
\hline Succión digital y bruxismo & 71 & 4.9 \\
\hline Otros hábitos & 10 & \\
\hline Ninguno & & \\
\hline
\end{tabular}

En relación con la presencia de maloclusiones, las más predominantes fueron la mordida profunda y la mordida abierta anterior. Sin embargo, no se observaron maloclusiones en el 50,4\% de los pacientes (tabla 4).

Tabla 4. Tipos de maloclusiones presentes

\begin{tabular}{|c|c|c|}
\hline Tipos de maloclusiones & No de pacientes & $\%$ \\
\hline Mordida abierta anterior & 29 & 14.3 \\
\hline
\end{tabular}




\begin{tabular}{|l|c|c|}
\hline Mordida profunda & 32 & 15.8 \\
\hline \begin{tabular}{l} 
Mordida abierta $\begin{array}{l}\text { anterior y mordida } \\
\text { cruzada posterior } \\
\text { unilateral }\end{array}$ \\
\hline Otras maloclusiones
\end{tabular}$\quad 33$ & 2.9 \\
\hline Ninguna & 102 & 16.3 \\
\hline
\end{tabular}

En los niños con lactancia materna exclusiva, disminuyeron considerablemente las mal oclusiones (20,0\%) en comparación con los alimentados con lactancia materna combinada $(59,2 \%)$.

\section{Discusión}

Desde el nacimiento, el bebé necesita adaptarse a un mundo nuevo: el extrauterino, y para ello depende del cuidado ofrecido por sus padres u otros adultos responsables. Así, cada niño nace con una conducta heredada que lo impulsa a crecer, pero este proceso del crecimiento no puede tener lugar sin un ambiente facilitador, sobre todo, al comienzo, cuando prevalece una situación de dependencia absoluta 9 .

Los resultados confirman la relación directa entre la lactancia materna exclusiva y la prevención de distintos hábitos bucales deformantes; resultados que coinciden con los referidos por Carrasco et al ${ }^{10}$.

Diversos autores plantean que la alimentación al seno materno por 6 meses o más constituye un factor de protección de las maloclusiones dentales ${ }^{11}$.

Rondón R y colaboradores en su investigación encontraron 44 artículos publicados en Latinoamérica, desde el año 1991 hasta el año 2012, en los que se plantea el efecto de la lactancia materna sobre el aparato bucal y sus funciones, así como también los hábitos parafuncionales, alteraciones dento-bucomáxilo-facial asociadas a la carencia de lactancia materna y se destacan los beneficios que ofrece la alimentación natural en la salud bucodental del infante ${ }^{12}$. 
Según Vasconcelos, F. \& Cols. ${ }^{13}$ en su investigación concluyeron, que el fomento de una mayor duración de la lactancia materna contribuye a la reducción de la prevalencia de hábitos deformantes de succión y maloclusiones como la mordida abierta anterior.

Estos resultados son similares a los de Vergara Terrado et al, ${ }^{14}$ quienes encontraron que los que tuvieron lactancia materna exclusiva, presentaron menor número de maloclusiones. En el niño, el amamantamiento contribuye al desarrollo de los maxilares, pues además de ganar espacio para la ubicación de los dientes en la arcada, tiene la satisfacción del seno materno y no siente hambre en ningún momento, por lo cual no se chupará el dedo y la aparición de hábitos bucales deformantes será mucho menor.

A manera de colofón, los resultados obtenidos indican una relación entre el tiempo de amamantamiento menor a 6 meses y el desarrollo de hábitos deformantes. Tienen mayor riesgo de padecer estos hábitos, los niños que no recibieron lactancia o que la tuvieron solo durante periodos cortos.

\section{Referencias Bibliográficas}

1- OMS. Estrategia Mundial: La lactancia materna fundamental para la supervivencia infantil. 2004 [citado agosto 23] Disponible en: www.who.int/mediacentre/news/releases/2004/pr19/es

2- Widmalm SE, Christiansen RL, Gunn SM. Oral parafunctions and temporomandibular disorder risk factors in children. Cranio 1995; 13:242-246

3- Navarro Nápoles J, Duharte Escalante A. La lactancia materna y su relación con los hábitos bucales Incorrectos. MEDISAN 2003; 7(2):17-21

4 López Méndez Y, Arias M, Valle Zelenko O del. La lactancia materna en la prevención de anomalías dentofaciales. Rev Cubana Ortod 1999; 14(1):32-7 
5- Carias D, Velázquez G, Cioccia AM, Piñero D, Inciarte H, Hervia P. Variaciones temporales en la composición y aporte de macronutrientes y minerales en leches maternas de mujeres venezolanas. Arch Latinoam Nutr 1999; 47(2): 110-7

6- Pipa-Vallejo A, Cuerpo-García P, López-Arranz E, González-García M, Pipa-Muñiz I, Acevedo-Prado A. Prevalencia de maloclusión en relación con hábitos de succión no nutritivos en niños de 3 a 9 años en Ferrol. Av Odontoestomatol.2011; 27 (3): 137-45

7- Herrera D, Belmonte S, Herrera E. Alteraciones del desarrollo maxilofacial. Prevención de la maloclusión. Arch Argent Pediatr. 2006; 104(1): 75-9

8- Morales-Chávez MC, Stabile-Del Vechio RM. Influencia de la lactancia materna en la aparición de hábitos parafuncionales y maloclusiones: estudio transversal. Univ Odontol. 2014 [citado 2017 agosto 23]; 33 (71). Disponible en: http://dx.doi.org/10.11144/Javerianuo3371.ilma

9- Ortega Valdés G. Ventajas de la lactancia materna para la salud bucodental. Notas breves. Rev Cubana Ortod. 1997;13(1):53-54

10- Carrasco M, Villena R, Pachas F, Sánchez Y. Lactancia materna y hábitos de succión nutritivos y no nutritivos en niños de 0-71 meses de comunidades urbano marginales del cono norte de Lima. Rev Estomatol Herediana. 2009 [citado 2017 agosto 23]; 19 (2):83-90. Disponible en: http://www.upch.edu.pe/faest/publica/2009/vol19_n2http /vol19_n2_09_art2.pdf

11- Blanco-Cedres Lucila, Guerra María E, Rodríguez Sebastián. Lactancia materna y maloclusiones dentales en preescolares de la Gran Caracas. Acta Odontológica Venezolana. 2007. 45 (2): 34-9. Disponible https://www.actaodontologica.com/ediciones/2007/2/lactancia_materna_maloclusiones_dent ales.asp 
12- Rondón, Rosa, Zambrano, Gabriel, Guerra, María Elena. Relación de la lactancia materna y el desarrollo Dento-Buco-Máxilo-Facial: Revisión de la literatura latinoamericana. Rev Latinoamericana de Ortodoncia y Odontopediatria .2012. 2014 [citado 2017 agosto 23] Disponible en: https://www.ortodoncia.ws/publicaciones/2012/art-19/

13- Vasconcelos, F; De Lima, A; Vilela, M; Brito, A; Tornisiello, C; Rosenblatt: Non-nutritive sucking habits, anterior open bite and associated factors in Brazilian children aged 30-59 months. Braz. Dent. 2011. 22(2)

14 Vergara Terrado Rolando Oscar, Barrueco Botiel Ligia Beatriz, Díaz del Mazo Lizel, Pérez Aguirre Evelyn, Sánchez Ochoa Thailen. Influencia de la lactancia materna sobre la aparición de maloclusiones en escolares de 5 a 6 años. MEDISAN. 2014 [citado 2017 agosto 23]; 18(8): 1064-1070. Disponible en: http://scielo.sld.cu/scielo.php?script=sci_arttext\&pid=S1029$30192014000800005 \& \operatorname{lng}=\mathrm{es}$ 